\title{
CONTRIBUTIONS TO THE BOHR TOPOLOGY BY W. W. COMFORT
}

\author{
SALVADOR HERNÁNDEZ, DIETER REMUS, AND F. JAVIER TRIGOS-ARRIETA
}

\begin{abstract}
The important rôle that W. W. Comfort played in the study of the Bohr topology is de-
\end{abstract} scribed.

\section{INTRODUCTION}

William Wistar Comfort II died on November 28, 2016 in Middletown, Connecticut, at age 83, following a brief illness. The three co-authors of the present article were lucky to be among his many friends, students and colleagues. This article is not intended to be a complete account of Wis' many contributions to mathematics. We will just touch upon a branch of mathematics that was very dear to him, the Bohr topology. For biographical accounts, the interested reader can access the interview of Wis by N. Hindman [56], or Wis' obituary [8, 9]. One of us, Remus, wrote an article on Wis' contributions to topological groups [74].

The loss of Wis has been very difficult to bear for us. Not only we, not only his family, not only mathematics, but the world has lost a man whose integrity, gallantry, and humility should set an example for what a decent human being should be.

\section{Preliminaries}

Let $G$ be a topological group. Then there exists a compact group $b G$ and a continuous homomorphism $b: G \longrightarrow b G$ with the following property: if $f: G \longrightarrow K$ is a continuous homomorphism from $G$ into a compact group $K$, there exists a continuous homomorphism $\bar{f}: b G \longrightarrow K$ such that $f=\bar{f} \circ b . b G$ is called the Bohr compactification of $G$, and $b$ the corresponding Bohr homomorphism. A topological group $G$ is said to be a maximally almost periodic (MAP) group if the Bohr homomorphism $b: G \longrightarrow b G$ is injective. This means: Whenever $g \in G$ and $g \neq 0 \in G$, there exists a continuous homomorphism $\phi$ of $G$ into a compact group, say $K_{g}$, such that $\phi(g) \neq 0 \in K_{g}$. The very important torus group $\mathbb{T}$ is defined as in [54] (§1), but sometimes we identify it with the group $([0,1),+\bmod 1)$. An Abelian topological group is MAP if and only if whenever $g \in G$ and $g \neq 0 \in G$, there exists a continuous homomorphism $\phi: G \longrightarrow \mathbb{T}$ such that $\phi(g) \neq 0 \in \mathbb{T}$. For more details on the Bohr compactification see [55]. It is a consequence of the Gel'fand-Raǐkov Theorem that locally compact Abelian groups (LCAGs) are MAP. A topological group $G$ is said to be precompact if given any open subset $U$ of $G$, there is a finite subset $F$ of $G$ such that $G=U F$. A Hausdorff precompact topological group is called totally bounded. It is a theorem of A. Weil that if a group is totally bounded, there exists a compact group $\bar{G}$ in which $G$ lives densely. We refer to $\bar{G}$ as the Weil completion of $G$ and it is unique up to a topological isomorphism ([92] and [93]). If $G$ is an Abelian topological group, we set $G^{+}:=b[G]$, and when $G$ is discrete, we use the special symbol $G^{\#}$ instead of $G^{+}$. Notice then that the topology of $G^{\#}$ is the finest totally bounded group topology that any abstract group $G$ accepts. For an Abelian MAP $G$, we will denote by $\widehat{G}$ the set of continuous homomorphisms $\phi: G \longrightarrow \mathbb{T}$, which we will refer to also as the characters of $G$. $\widehat{G}$ becomes a topological group by defining $\left(\phi_{1} \phi_{2}\right)(g):=\phi_{1}(g) \phi_{2}(g) \in \mathbb{T}$ whenever $g \in G$ and equipping it with the compact-open topology. It is a direct implication from the Comfort-Ross Theorem [25] (see $\S 3$ below), that the topology of $G^{+}$is the weakest topology that makes the elements of $\widehat{G}$ continuous. When $G$ is

Date: February 25, 2019.

2010 Mathematics Subject Classification. Primary: 22B05; Secondary: 54H11.

Key words and phrases. group, Abelian group, characters, weak topologies, Weil completion, Bohr compactification, Bohr topology, locally compact group, maximally almost periodic group, minimally almost periodic group, compactness, pseudocompactness, realcompactness. 
locally compact, $\widehat{G}$ is also locally compact. It is a theorem of I. Glicksberg [47], that for $G$ locally compact and Abelian, $G$ and $G^{+}$have exactly the same compact subspaces. We call this result Glicksberg's theorem. Several alternate proofs and generalizations of Glicksberg's theorem have been given (see [31], [38], [41], [42], [57], [64], [65](Chapters 41 and 42), and [75]).

Historical developments concerning almost periodic functions and Bohr compactifications are described in [1], [61] (pp. 222-235) and [66] (3C, pp. 45-51). For undefined terms in this article, we refer the reader to the monographs [11], [36], and [54].

\section{The beginnings. Comfort and Ross 1964 [25], Comfort and Saks 1973 [26], Comfort 1984 [11], Comfort 1985 [10], Comfort And Robertson 1987 [24]}

Let $\mathcal{P K}(G)$, resp. $\mathcal{B}(G)$, denote the lattice of all precompact (not necessarily Hausdorff) group topologies, resp. the set of all totally bounded group topologies on a group $G$. Wis and K. A. Ross were the first who considered $\mathcal{B}(G)$. In 1964 they described it for Abelian groups.

Theorem 3.1. [25] Let $G$ be an Abelian group, and let $G^{*}$ be the group of all homomorphisms of $G$ into the torus $\mathbb{T}$. For each $\tau \in \mathcal{B}(G)$ let $\psi(\tau)$ be the group of all continuous characters of $(G, \tau)$. Then $\psi$ is a bijection between $\mathcal{B}(G)$ and the set of the point-separating subgroups of $G^{*}$.

By using Theorem 3.1 the second author proved in [77] that for every Abelian group $G$ the lattice $\mathcal{P K}(G)$ is isomorphic to the lattice of all subgroups of $G^{*}$.

Let $G$ be a group, and let $\tau_{G}^{d}$ be the discrete topology on $G \cdot \mathcal{L} \mathcal{A}\left(b G, b \tau_{G}^{d}\right)$ stands for the lattice of all closed normal subgroups of $\left(b G, b \tau_{G}^{d}\right)$, the Bohr compactification of $\left(G, \tau_{G}^{d}\right)$, and $e$ is the identity of $b G$. Then Theorem 3.1 was generalized by the second author in [77] (3.7) (see also [71], Lemma 2), as follows.

Theorem 3.2. For every group $G$ there exists a function $\Phi: \mathcal{P K}(G) \rightarrow \mathcal{L} \mathcal{A}\left(b G, b \tau_{G}^{d}\right)$ which is an antiisomorphism of lattices. If $\left(G, \tau_{G}^{d}\right)$ is maximally almost periodic, then $\Phi$ maps the totally bounded group topologies on those closed normal subgroups $N$ of $\left(b G, b \tau_{G}^{d}\right)$, satisfying $b[G] \cap N=\{e\}$.

In [37], a different extension of Theorem 3.1 to non-Abelian groups was given by M. V. Ferrer and the first author.

J. O. Kiltinen [58] proved in 1974 that every infinite Abelian group $G$ admits exactly $2^{2^{|G|}}$-many Hausdorff group topologies. Later on K.-P. Podewski [67] reproved this result independently by different methods. For non-Abelian groups without a nondiscrete Hausdorff group topology see [16], pp. 89-91. By using Theorem 3.1, the result of Kiltinen was independently improved in 1982 by S. Berhanu, Wis and J. D. Reid [6], and the second author [77] and [72].

Theorem 3.3. Every infinite Abelian group $G$ admits exactly $2^{2^{|G|}}$-many totally bounded group topologies.

Recently I. K. Babenko and S. A. Bogatyi [3] have reproved Theorem 3.3. Their main tool is also Theorem 3.1. Surprisingly, they did not mention the much earlier work of Berhanu et al. and of the second author.

For an extension of Theorem 3.3 to non-Abelian groups see [71], [73], [78] and [21], where Theorem 3.2 is used as a main tool. In [20], the Peter-Weyl theorem and unitary representations are applied. The number of pseudocompact group topologies on compact Abelian groups is studied in [19] and in [12], with the help of Theorem 3.1. For arbitrary compact groups see [22], Sections 5.2 and 8.2.

Wis, H. Szambien and the second author used in [23] Bohr compactifications of topological rings and a modification of Theorem 3.2 (see [23], Theorem 2.6) to study the number of pseudocompact ring topologies on compact rings. Let $\alpha$ be a cardinal considered as a (limit) ordinal. Then $\operatorname{cf}(\alpha)$ is the least cardinal $\beta$ such that there is a function $f: \beta \longrightarrow \alpha$ which is cofinal (unbounded) in $\alpha$. If $X$ is a topological space, $w(X)$ denotes its weight (cf. [36] (1.1)). Among other things, the following holds.

Theorem 3.4. ([23], Theorem 3.6) Let $(R, \tau)$ be a compact ring for which $\alpha:=w(R, \tau)$ satisfies $\operatorname{cf}(\alpha)>$ $\omega$. Assume $w(R / J(R))=\alpha$, where $J(R)$ is the Jacobson radical of the ring $R$. Then there are exactly $2^{2^{|R|}}$-many pseudocompact ring topologies on $R$ which are finer than $\tau$. In particular this holds if $R$ is a semisimple ring. 
Berhanu et al. [6] started the investigation of the poset $\mathcal{B}(G)$ for Abelian groups $G$ by computing cardinal invariants of $\mathcal{B}(G)$ like width, height and depth. For more on the subject see [74], Section 1.

In $2016 \mathrm{Wis}$ and the second author stated several problems in [22]. Concerning the number of pseudocompact group topologies the following is still open.

Problem 3.5. (a)([22], Problem 5.2.7(b)). Let $(K, \tau)$ be a nonmetrizable compact connected group. Are there $2^{2^{|G|}}$-many pseudocompact group topologies on $G$ which are finer than $\tau$ ?

(b)([22], Problem 8.2.11(b)). Let $(K, \tau)$ be a nonmetrizable profinite group. Are there $2^{2^{|G|}}$-many pseudocompact group topologies on $G$ which are finer than $\tau$ ? (A compact group is profinite if and only if it is totally disconnected.)

Wis and V. Saks [26] noted that the compact group topology of the real special orthogonal group $\mathrm{SO}(3, \mathbb{R})$ is the finest totally bounded group topology on this group. It was Lew Robertson who pointed out to both authors that this follows from a result of B. L. van der Waerden [88]. This led Wis [10] to the following

Definition 3.6. A compact group is called a van der Waerden group (vdW-group) if every homomorphism to a compact group is continuous.

It can be easily seen (cf. [26], pp. 39-40) that a compact group $G$ is a vdW-group if and only if its topology is the finest totally bounded group topology on $G$. By van der Waerden's result every compact connected semisimple Lie group is a vdW-group. (In the special case of $\mathrm{SO}(3, \mathbb{R})$ Wis and L. C. Robertson [24] gave a transparent, nearly elementary proof). For more about vdW-groups see [50], [64], [74], and [94].

A compact group $G$ is called tall if for every positive integer $n$ the set of pairwise nonequivalent continuous irreducible unitary representations of $G$ whose dimensions do not exceed $n$ is finite. It follows from [64] that every vdW-group is tall and hence metrizable. There are profinite tall groups which are not vdW-groups (see [50], Example 7.2). Wis and the second author made the following conjecture many years ago. In 2014 it was stated in print for the first time in the paper [80] of A. I. Shtern.

Conjecture 3.7. A compact connected group is a vdW-group if and only if it is tall.

It is well-known that the conjecture holds for compact Lie groups. In the last telephone conversation with the second author, shortly before his death, Wis said that Conjecture 3.7 was one of his favorite problems during the last ten years. It is still unsolved.

In [23] van der Waerden rings are defined in the class of compact rings in an obvious way. Among other things, a structure theorem for semisimple vdW-rings was given (see [23], Theorem 4.13). Recently M. Ursul and the second author [76] have made significant progresses concerning the structure of vdWrings .

Probably, the first results obtained by Wis about Bohr topologies on Abelian groups were with Saks in [26]: (1) Subgroups of $G^{\#}$ are all closed, (2) if infinite, $G^{\#}$ is never pseudocompact, and (3) a product of groups $G_{i}^{\#}$ has its finest totally bounded group topology if and only if it is a finite product. Property (1) was eventually generalized to the fact that closed subgroups of a LCAG $G$ remain closed as subspaces of $G^{+}$[85]. It was Lew Robertson who made the authors of [85] aware of Glicksberg's result [47], which led Wis to show that only the finite sets were the pseudocompact or countably compact subspaces of any $G^{\#}[27]$. This fact was eventually generalized in [84] to the fact that no pseudocompact subspaces are created for a LCAG to the passage to its Bohr topology.

Wis' contribution to the Handbook of General Topology [11], published in 1984, outlines the importance that he gave to the Bohr topology. Section 4 can be considered an introduction to the Bohr topology for a beginning student with basic knowledge in general topology and group theory, as it yields most of the tools necessary for its study. The Bohr topology is specifically defined in 4.26 and $G^{+}$for a LCAG $G$ is given in 4.27 (with $G^{+}=\zeta[G]$ ).

\section{The study of the maximal totally bounded group topology on Abelian groups [27]}

The late E. van Douwen sent in the mid-eighties two letters to Wis [90] and a preprint of [89], in which he stated a few unpublished results and asks some questions. During the Fall semester of 1987, Wis gave a course at Wesleyan University on topological groups which the third author attended as a 
student. Wis presented these results and questions to the class, and they became the project for the doctoral thesis [87] and eventually for the articles [27], [85] and [84]. Van Douwen, for example, shows in [89] that $G^{\#}$ is zero-dimensional. This result was independently re-discovered by Lew Robertson and the third author, and was published in [27]. Since D. B. Shakhmatov had shown that, for totally bounded groups, zero-dimensionality and strong zero-dimensionality coincide [79], it follows that $G^{\#}$ is strongly zero-dimensional, the answer to an original question raised by van Douwen in one of his letters to Wis (see [51] for further information about dimension and the Bohr topology). The Bohr topology has also been applied to prove the existence of Abelian groups with non-normal precompact topologies (see the article [86] of the third author), answering related questions of A. A. Markoff [63] and van Douwen [89].

\section{The Bohr compactification of a LCA group modulo a metrizable subgroup [28]}

If $N$ is a closed subgroup of $b G$, the authors of [28] say thay $N$ preserves compactness if a subspace $A$ of $G$ satisfies that $\phi[b[A]]$ is compact in $b G / N$ if and only if $A\left(b^{-1}\left[G^{+} \cap N\right]\right)$ is compact in $G$, where $\phi: b G \longrightarrow b G / N$ denotes the natural quotient map. When the closed metrizable subgroups of $b G$ preserve compactness, we say that $G$ strongly respects compactness. In [28] it is shown that LCAGs strongly respect compactness. This result generalizes Glicksberg's theorem [47] by taking $N:=\left\{0_{b G}\right\}$ above. In the same article, the authors show that the result can fail if $N$ is not metrizable, even if $N \cap G^{+}=\left\{0_{G^{+}}\right\}$; but they also show that the result may still hold for non-metrizable closed subgroups $N$ of $G$.

The case $G$ discrete turned out to be more difficult than expected and a misleading and most likely incorrect proof was printed in [28]. The first author and Jorge Galindo noticed the mistake and informed the authors of [28] about it. Eventually, a sound proof of the case $G$ discrete was found and subsequently published as a correction in [29].

The paper [28] then discusses the interplay between $k$-topologies and the Bohr topology. Recall that a $k$-space $X$ is one in which every set $U$ satisfying that $U \cap K$ is an open subset of every compact subspace $K$ of $X$ is itself open in $X$. If $Y$ is a space, there exists a $k$-space $X$ and a continuous bijection $f: X \longrightarrow Y$ such that $K$ compact in $Y \Longrightarrow f^{-1}[K]$ compact in $X$, and one shows that, up to a homeomorphism such $X$ is unique. We then write $k Y=X$. W. F. LaMartin [?] (2.1) showed that $k\left(\mathbb{R}^{\alpha}\right)$ is not a topological group whenever $\alpha>\aleph_{0}$, hence the $k$-ification of a topological group need not be a topological group. Glicksberg's theorem [47] implies then that if $G$ is a LCAG, then $G=k G^{+}$. The main result of [28] yields a totally bounded group $H$ with $G:=k H$ being a locally compact Abelian group, yet $H \neq G^{+}$. The characterization of those totally bounded groups $H$ such that $G:=k H$ is a locally compact Abelian group, and $H=G^{+}$, was posed as Question 4.3, studied by J. Galindo in [43], and recently solved by the first and last authors in the paper [53].

The rest of [28] consists of constructing examples of LCAGs $G$ and closed, non-metrizable subgroups $N$ of $b G$ preserving compactness. Paramount for this is lemma 3.11, a very interesting result in itself: Assume that $G$ is a discrete Abelian group, $N$ a closed subgroup of $b G$ with $N \cap G=\{0\}$, and $\lambda$ the

Haar measure of $\widehat{G}$. Let $\mathbb{A}(\widehat{b G}, N):=\{\chi \in \widehat{b G}: \chi[N]=\{0\}\}$, i.e., $\mathbb{A}(\widehat{b G}, N)$ is the annihilator of $N$ in $\widehat{b G}$. Suppose that either $\left\{\chi_{\mid G}: \chi \in \mathbb{A}(\widehat{b G}, N)\right\}$ is not $\lambda$-measurable in $\widehat{G}$, or $\lambda\left(\left\{\chi_{\mid G}: \chi \in \mathbb{A}(\widehat{b G}, N)\right\}\right)>0$. Denote by $\phi: G \longrightarrow b G / N$ the natural map. If $G$ is countable or $\phi[H]$ is closed in $b G / N$, whenever $H$ is a subgroup of $G$, then $N$ preserves compactness. So, here it is a connection between preserving compactness and the Haar measure of the character group, that has been exploited by several mathematicians. In another direction, the class of groups that strongly preserve compactness has been considered by different authors for Abelian and non-Abelian groups. We refer to [39], [45] and the references therein.

The article [28] concludes with a number of questions, some of them already answered but others still open. We already mentioned the solution to Question 4.3, and in the contribution [53] the first and last authors show that there is a MAP group $G$ not strongly respecting compactness, yet having all closed metrizable subgroups $N$ of $b G$ with $N \cap G^{+}=\{0\}$, preserving compactness, answering hence Question 4.1 of $[28]$.

\section{Realcompactness of the Bohr topology [14]}

In the article [89], van Douwen asked whether $G^{\#}$ is realcompact when $|G|$ is not Ulam-measurable, as he knew that $G^{\#}$ containes a closed discrete $C$-embedded subspace of cardinality $|G|$, hence if $G^{\#}$ is realcompact, then $|G|$ cannot be Ulam-measurable. In [14] the authors prove that $G^{\#}$ is realcompact if 
and only if $|G|$ is not Ulam-measurable. First they give an alternative proof of the sufficiency. Necessity is achieved as follows: If $G$ is a LCAG, denote by $\widetilde{G}^{b G}$ the $\mathcal{G}_{\delta}$-closure of $G^{+}$, i.e., $p \in b G$ belongs to $\widetilde{G}^{b G}$ if and only if every $\mathcal{G}_{\delta^{-}}$set in $b G$ containing $p$ hits $G^{+}$. If we consider $b G \subseteq \mathbb{T}^{\widehat{G}}$, then $p \in b G$ belongs to $\widetilde{G}^{b G}$ if and only if $p$ is continuous on every countable subset of $\widehat{G}$. Since one always has $|\widehat{G}| \leq 2^{|G|}$, notice then that if $|G|$ is not Ulam-measurable, then $|\widehat{G}|$ cannot be Ulam-measurable either. It is then immediate from a result of Varopoulos [91] that every element of $b G$ which is continuous on every countable subset of $\widehat{G}$ is in fact continuous on $\widehat{G}$, so the above gives $\widetilde{G}^{b G}=G^{\#}$. Thus $G^{\#}$, being $\mathcal{G}_{\delta}$-closed in the (real)compact space $b G$, is itself realcompact.

The article [14] gives as well a characterization for LCAGs $G$ when $G^{+}$is realcompact. For example, this is equivalent to $G$ being realcompact, to $G^{+}$being topologically complete, and to $|G / H|$ not being Ulam-measurable, whenever $H$ is a $\sigma$-compact open subgroup of $G$. Another characterization achieved in [14] is when for a LCAG $G, G^{+}$is hereditarily realcompact. It follows that this happens precisely when the points of $G^{+}$are $\mathcal{G}_{\delta}$-s, or equivalently, when $G$ is metrizable and $|G| \leq \mathfrak{c}$. The authors show as well that for every LCAG $G$, every closed subgroup $H$ of $G$ is $C$-embedded in $G^{+}$; indeed when $G$ is discrete, then every continuous function $f: H^{+} \longrightarrow S$ with $S$ completely metrizable extends continuously to some $\bar{f}: G^{+} \longrightarrow S$.

The article finishes with several questions, many of them still unanswered.

\section{The maximal totally bounded Group topology: Homeomorphisms and Retracts [15]}

A remarkable feature of the Bohr topology is that, except for the standing Abelian hypothesis, basically all results around concerning \#-groups make no use whatever of specific algebraic properties and the arguments turn strictly on combinatorics related to the cardinality of the groups in question. This fact probably led van Douwen [89] to pose the following question:

Question 7.1. If the groups $G_{1}$ and $G_{2}$ have the same cardinality, must the spaces $G_{1}^{\#}$ and $G_{2}^{\#}$ be homeomorphic?

In a preliminary response in the positive direction, the third author noticed [87] (6.33 and 6.36) that if $G$ contains a subgroup $H$ of index $n \in \mathbb{N}$ such that $G^{\#}$ and $H^{\#}$ are homeomorphic, then $G^{\#}$ is homeomorphic to $G^{\#} \times \mathbb{Z}_{n}$, where $\mathbb{Z}_{n}$ stands for the discrete cyclic group of order $n$, thus showing that non-isomorphic groups of arbitrary infinite cardinal do exist with homeomorphic \#-spaces. Using the fact that every nondiscrete countable infinite homogeneous space is homeomorphic to each of its nonempty clopen subsets, J. E. Hart and K. Kunen [49], generalized the third author's result, showing for every infinite group $G$ that $G^{\#}$ and $G^{\#} \times D$ are homeomorphic for every finite or countably infinite discrete space $D$. Nevertheless, K. Kunen [59], and independently D. Dikranjan and S. Watson [34], have given examples of torsion groups with the same cardinality yielding nonhomeomorphic \#-spaces. Thus Question 7.1 is solved in the negative. Much remains unknown, however, even among groups of countable cardinality (see [30] and the references therein). For instance, the arguments of [59] and [34] leave (for example) the following questions untouched: Which if any of the spaces $\mathbb{Z}^{\#},(\mathbb{Z} \times \mathbb{Z})^{\#}$, $(\operatorname{div}(\mathbb{Z}))^{\#},(\operatorname{div}(\mathbb{Z}) / \mathbb{Z})^{\#},((\operatorname{div}(\mathbb{Z}) / \mathbb{Z}) \times \mathbb{Z})^{\#},\left(\oplus_{\omega} \mathbb{Z}\right)^{\#},\left(\oplus_{\omega}\{0,1\}\right)^{\#}$ are homeomorphic? $(\operatorname{div}(H)$ stands for the divisible hull of an Abelian group $H$.) It seems appropriate, accordingly, to replace Question 7.1 with the following (imperfectly posed) open-ended question.

Question 7.2. How do algebraic properties of the groups $G$ affect the homeomorphism classes of the spaces $G^{\#}$ ?

In relation to the question above for the specific groups $\mathbb{Z}^{\#}$ and $(\mathbb{Z} \times \mathbb{Z})^{\#}$, in $[5] \S 4$, Wis pointed out that $\mathbb{Z}$ and $\mathbb{Z} \times \mathbb{Z}$ do admit many pairwise homeomorphic non-discrete topologies. It is known [25] (see Theorem 3.1 above) for each infinite subgroup $A$ in $G^{*}$ that $w\left(G, \tau_{A}\right)=|A|$ and that the elements of $A$ are exactly the $\tau_{A}$-continuous homomorphisms from $G$ to $\mathbb{T}$, so distinct subgroups of $G^{*}$ induce distinct group topologies on $G$. When $|G|=\omega$ and $A$ is countable, the space $\left(G, \tau_{A}\right)$ is a countably infinite metrizable space without isolated points, hence according to a familiar theorem of Sierpiński [81], homeomorphic to $\mathbb{Q}$ in its usual topology. Thus $\mathbb{Z}$ and $\mathbb{Z} \times \mathbb{Z}$ admit $\mathfrak{c}$-many distinct metrizable topological group topologies (of the form $\tau_{A}$ ), each homeomorphic to $\mathbb{Q}$. However, this observation does not apply to the Bohr topology, since when $|G|=\omega$ the space $G^{\#}$ has weight equal to $\mathfrak{c}$. 
When given groups $G$ and $H$, Kunen [59] writes $G \sim H$ in case there are subgroups $G_{1}$ of $G$, and $H_{1}$ of $H$, each of finite index, such that $G_{1}$ and $H_{1}$ are isomorphic. He remarked that if $G \sim H$ then $G^{\#}$ and $H^{\#}$ are homeomorphic, and he asked if the converse holds. It is proven in [15] that the spaces $(\operatorname{div}(\mathbb{Z}))^{\#}$ and $((\operatorname{div}(\mathbb{Z}) / \mathbb{Z}) \times \mathbb{Z})^{\#}$ are homeomorphic, simultaneously contributing to Question 7.2 above and showing that the answer to Kunen's question is "not always".

If $H$ has finite index in $G$, then $H^{\#}$ is clopen in $G^{\#}$, hence it is a retract of $G^{\#}$. This phenomenon led van Douwen [89] to pose also the following question.

Question 7.3. Is every subgroup $H$ of a group $G^{\#}$ a retract of $G^{\#}$ ?

Article [15] also contributes towards Question 7.3 by identifying large classes of groups which are retracts wherever they are embedded. In this approach the following definition is essential.

Definition 7.4. Let $H$ be a closed subgroup of a (not necessarily Abelian) topological group $G$, and let $\pi: G \longrightarrow G / H$ be the natural map.

(a) A continuous cross section for $G / H$ is a continuous map $\Gamma: G / H \longrightarrow G$ such that $\pi \circ \Gamma=\mathrm{id}_{\mid G / H}$;

(b) if $G / H$ has a continuous cross section, then $H$ is a ccs-subgroup of $G$.

It is proved that if $H$ is a ccs-subgroup of a topological group $G$, then $G$ is homeomorphic to $(G / H) \times H$ and $H$ is a retract of $G$. Furthermore $H^{\#}$ is a ccs-subgroup of every enveloping \#-group if and only if $H^{\#}$ is a ccs-subgroup of $(\operatorname{div}(H))^{\#}$. Since every finitely generated group is a ccs-subgroup of every enveloping \#-group, it is obtained that $(\operatorname{div}(\mathbb{Z}) / \mathbb{Z})^{\#} \times \mathbb{Z}^{\#}$ is homeomorphic to $(\operatorname{div}(\mathbb{Z}))^{\#}$ and $\mathbb{Z}^{\#}$ is a retract of $(\operatorname{div}(\mathbb{Z}))^{\#}$. A topological group $H$ is an absolute ccs-group $(\#)$ if $H$ is a ccs-subgroup in every group of the form $G^{\#}$ containing $H$ as a (necessarily closed) subgroup. Finally, using techniques and arguments drawn from Kunen [59], an example of a group which is not an absolute ccs-group(\#) is given. Nevertheless, Question 7.3 is still open.

\section{Character groups of Dense subgroups [17]}

If $H$ is a dense subgroup of a topological group $G$, then because of the uniform continuity of the elements of $\widehat{H}$, these can be extended uniquely to elements in $\widehat{G}$, i.e., as groups, $\widehat{H}$ and $\widehat{G}$ are isomorphic. But as topological groups there is no reason for them to be topologically isomorphic. Surely, a compact subspace of $H$ is still compact in $G$, but singletons in $G \backslash H$ are, for example, compact subspaces of $G$ not in $H$. Hence, the restriction map to $H, r_{\mid H}: \widehat{G} \longrightarrow \widehat{H}$ is continuous but does not have to be open. However, when $G$ is metrizable, $r_{\mid H}$ is indeed open and therefore, a topological isomorphism, as proven by L. Aussenhofer [2] and M. J. Chasco [7], independently. In her doctoral thesis [70], S. U. Raczkowski called a dense subgroup $H$ of $G$ determining if $r_{\mid H}$ is a topological isomorphism, and $G$ determined if all its dense subgroups were determining. Under this nomenclature, all metrizable groups are determined. In the article [17], and drawing from [70], it is pointed out that if $G$ is a non-compact, metrizable LCAG, then with the aid of Glicksberg's Theorem [47], $G^{+}$is determined, hence yielding many non-metrizable, non-compact determined groups. In fact, if $G$ is locally bounded (i.e., its Weil completion is locally compact), then $G$ is determined if and only if $G^{+}$is determined. On the other hand, if $G$ is a LCAG, then $G$ non-compact implies that $G^{+}$does not determine $b G$. It is also shown in [17] that if $G$ is a compact group with $w(G) \geq \mathfrak{c}$, then $G$ is not determined, and under $\mathrm{CH}$, that a compact group is determined if and only if it is metrizable. Eventually, S. Macario along with the first and third authors [52] removed the $\mathrm{CH}$ requirement. Further progress on this question has been accomplished by several authors. D. Dikranjan and D. Shakhmatov proved in [32] that for every infinite compact Abelian group $G$ we have that $w(G)=\min \{|D|: D$ is a subgroup of $G$ that determines $G\}$. Finally, M. V. Ferrer, the first author and V. Uspenskij [40] extended the main result of Wis et al [17] and the first author et al [52] to not necessarily Abelian compact groups. That is, a compact group $G$ is determined if and only if it is metrizable.

\section{Maximum number of totally bounded group topologies on Abelian groups, with and WITHOUT CONVERGENT SEQUENCES [18]}

Not long after the invention of the Stone-Čech compactification $\beta X$ of the Tychonoff space $X$, it was noted that $\beta(\omega)$ contains no nontrivial convergent sequences. Hence, B. Efimov [35] posed in 1969 a question which in ZFC remains unsolved today: Does every compact Hausdorff space contain either a copy 
of $\beta(\omega)$ or a nontrivial convergent sequence? The paper [18] is concerned with topological groups within this contex: Given a class $\mathcal{C}$ of topological groups, does every group in $\mathcal{C}$ contain a nontrivial convergent sequence? (a) Every infinite compact group $K$ contains topologically a copy of the generalized Cantor space $\{0,1\}^{w(K)}$, hence having a convergent non-trivial sequence, a result to which Šapirovskiĭ, Gerlits and Efimov have contributed; (b) Assuming GCH, V. I. Malykhin and L. B. Shapiro [62] have shown that every totally bounded group $G$ with $w(G)<w(G)^{\omega}$ contains a nontrivial convergent sequence; (c) S. U. Raczkowski [69] and [70], and others [4], [95], [96] have shown that for every suitably fast-growing sequence $\left\langle x_{n}\right\rangle$ in $\mathbb{Z}$ there is a totally bounded group topology on $\mathbb{Z}$ with respect to which $x_{n} \rightarrow 0$. On the other hand, (d) recall that by Glicksberg's theorem [47], when a LCAG $G$ is given its associated Bohr topology, no new compact sets are created; in particular, as shown earlier by H. Leptin [60], the Bohr topology induced on a discrete Abelian group has no infinite compact subsets, in particular no nontrivial convergent sequences; (e) there are infinite pseudocompact topological groups containing no nontrivial convergent sequences [82]; see also [44] and [46] and the literature cited there for results in the same vein. (e) Perhaps the most celebrated unsolved related question is whether or not there exists in ZFC a countably compact topological group with no nontrivial convergent sequences. Many examples are known in augmented axiom systems. For the interested reader, the introduction of [18] gives additional information on the subject.

It has been known for quite sometime, that every infinite Abelian group contains a subgroup of infinite countable index. Hence, if compact, it contains a non-Haar-measurable subgroup [54] (16.13 (c)). It has been noticed by K. R. Stromberg [83] that $\mathbb{R}$ contains a nonmeasurable subgroup of index $\mathfrak{c}$. The authors of [18] show then that every infinite compactly generated Abelian group $K$ has a family of $2^{|K|}$-many dense, nonmeasurable subgroups of index $|K|$. From this it follows that every infinite Abelian group $G$ admits a family of $2^{2^{|G|}}$-many totally bounded group topologies $\tau$ with no nontrivial convergent sequences. By very different methods it is shown that such $G$ has the same number of totally bounded group topologies $\tau$ in each of which some nontrivial sequence (fixed, and chosen in advance) does converge. In the obvious sense, these results are clearly optimal. An elementary cardinality argument shows that the various topological groups $(G, \tau)$ may be chosen to be pairwise nonhomeomorphic as topological spaces.

\section{Minimally almost periodic groups and the small subgroup generating Property [13]}

A topological group $G$ is called minimally almost periodic if the Bohr compactification of $G$ is the trivial group. For more details see [48], Chapter 2 and [13], Section 2. Wis became very interested in this class of groups since at least the 1990s (see [21], Section 4). In 2009 Frank Gould [48] finished a Ph.D. thesis under the supervision of Wis. It concerns certain classes of minimally almost periodic groups. Gould introduced the following definition which was motivated by the paper [68] of I. Prodanov.

Definition 10.1. ([48], Definition 3.1.1) A topological group $G$ has the small subgroup generating property or "SSGP" if it is Hausdorff and if every neighbourhood of the identity contains the union $\bigcup \mathcal{H}$ of a family $\mathcal{H}$ of subgroups of $G$ such that $\bigcup \mathcal{H}$ generates a dense subgroup of $G$.

It is easy to see that a topological group with SSGP is minimally almost periodic ([48], Theorem 3.1.2). The joint paper [13] of Wis and F. Gould derives from [48] and extends selected portions of that paper. In particular, they introduced the classes $\operatorname{SSGP}(n)$ for $0 \leq n<\omega$ as follows.

Definition 10.2. ([13], Definition 3.3) Let $G=(G, \tau)$ be a Hausdorff topological group. Then

(a) $G \in \operatorname{SSGP}(0)$ if $G$ is the trivial group,

(b) $G \in \operatorname{SSGP}(n+1)$ for $n \geq 0$ if for every neighbourhood $U$ of the identity there is a family $\mathcal{H}$ of subgroups of $G$ such that

(1) $\bigcup \mathcal{H} \subseteq U$,

(2) $H:=\overline{\langle\bigcup \mathcal{H}\rangle}$ is normal in $G$, and

(3) $G / H \in \operatorname{SSGP}(n)$.

Clearly, the classes SSGP and SSGP(1) are the same. Furthermore, by [13] every group from $\operatorname{SSGP}(n)$ is contained in $\operatorname{SSGP}(n+1)$ and is minimally almost periodic. Let $(G, \tau)$ be a Hausdorff topological group. We say that $\tau$ is a $\operatorname{SSGP}(n)$ topology if $(G, \tau) \in \operatorname{SSGP}(n)$. The following theorem 
shows that there are minimally almost periodic groups which do not belong to any class $\operatorname{SSGP}(n)$ for $n>0$.

Theorem 10.3. ([13], Theorem 3.13) A nontrivial Abelian group $G$ which is the direct sum of a finitely generated group and a finitely cogenerated group does not admit a $\operatorname{SSGP}(n)$ topology for any $n<\omega$.

In [13] Theorem 3.18 many Abelian groups are presented which admit a SSGP topology. By [13] Corollary 3.28 an Abelian Hausdorff topological group of bounded order is minimally almost periodic if and only if it belongs to the class SSGP. It is shown that the class $\operatorname{SSGP}(n)$ is stable under products, direct sums and quotients. The paper [13] contains very interesting results about the aforementioned classes. It is a pleasure to read the paper!

The study of topological groups belonging to $\operatorname{SSGP}(n)$ has been continued by D. Dikranjan and D. Shakhmatov [33], where considerable progress has been made by answering some of the questions posed in [13].

\section{Acknowledgement}

We thank the referees for constructive criticism. We also are grateful to the editors, Tony Hager and Jan van Mill, for the invitation to participate in this special edition. The third author also thanks Sophia Raczkowski for having carefully read an earlier version of this article.

\section{REFERENCES}

1. J. Andres, A.M. Bersani, and R.F. Grande, Hierarchy of almost-periodic function spaces, Rend. Mat. Appl., VII. Ser. 26 (2006), 121-188.

2. L. Aussenhofer, Contributions to the duality theory of abelian topological groups and to the theory of nuclear groups, Diss. Math. 384 (1999), 113 pages.

3. I. K. Babenko and S. A. Bogatyi, On topologies on the group $\left(\mathbb{Z}_{p}\right)^{\mathbb{N}}$, Topology Appl. 221 (2017), 638-646.

4. D. Barbieri, D. Dikranjan, C. Milan, and H. Weber, Answer to Raczkowski's questions on convergent sequences of integers, Topology Appl. 132 (2003), 89-101.

5. V. Bergelson and B. Rothschild, A selection of open problems., Topology Appl. 156 (2009), 2674-2681.

6. S. Berhanu, W. W. Comfort, and J. D. Reid, Counting subgroups and topological group topologies, Pacific J. Math. 116 (1985), 217-241.

7. M. J. Chasco, Pontryagin duality for metrizable groups, Archiv Math. 70 (1998), 22-28.

8. M. W. Comfort, W. Wistar Comfort (1933-2016), Topology and Appl. (this issue).

9. M. W. Comfort, W. Wistar Comfort (1933-2016), Notices of the AMS 64-3 (2017), 275-276.

10. W. W. Comfort, Compact groups: subgroups and extensions, Topology, Theory and Applications, 5th Colloq., Eger/Hung. 1983, Colloq. Math. Soc. János Bolyai 41, 183-198 (1985).

11. W. W. Comfort, Topological groups, Handbook of Set-Theoretic Topology (K. Kunen and J. E. Vaughan, eds.), Elsevier Science Publishers, B. V., Amsterdam, 1984, pp. 1143-1263.

12. W. W. Comfort and J. Galindo, Pseudocompact topological group refinements of maximal weight, Proc. Am. Math. Soc. 131 (2003), 1311-1320.

13. W. W. Comfort and F. R. Gould, Some classes of minimally almost periodic topological groups, Appl. Gen. Topol. 16 (2015), 141-165.

14. W. W. Comfort, S. Hernández, and F. J. Trigos-Arrieta, Relating a locally compact abelian group to its Bohr compactification, Adv. Math. 120 (1996), 322-344.

15. Cross sections and homeomorphism classes of Abelian groups equipped with the Bohr topology, Topology Appl. 115 (2001), 215-233.

16. W. W. Comfort, K.-H. Hofmann, and D. Remus, Topological groups and semigroups, Recent progress in general topology. Papers from the Prague Toposym 1991, held in Prague, Czechoslovakia, Aug. 19-23, 1991, Amsterdam: North-Holland, 1992, pp. 57-144.

17. W. W. Comfort, S. U. Raczkowski, and F. J. Trigos-Arrieta, Concerning the dual group of a dense subgroup, Proc. Ninth (2001) Prague Toposym (2002), 23-35, Topology Atlas, Toronto, 2002.

18. _ Making group topologies with, and without, convergent sequences, Appl. Gen. Topol. 7 (2007), 109-124.

19. W. W. Comfort and D. Remus, Pseudocompact refinements of compact group topologies, Math. Z. 215 (1994), 337-346.

20. I I I I tervals of totally bounded group topologies, Papers on general topology and applications. Papers presented at the 11th summer conference at the University of Southern Maine, Gorham, ME, USA, August 10-13, 1995, New York, NY: The New York Academy of Sciences, 1996, pp. 121-129.

21. _ L L L L chains of topological group topologies. — A continuation, Topology Appl. 75 (1997), 51-79.

22. N_ Non-abelian pseudocompact groups, Axioms 5(1) (2016), 17 pages.

23. W. W. Comfort, D. Remus, and H. Szambien, Extending ring topologies, J. Algebra 232 (2000), 21-47.

24. W. W. Comfort and L. C. Robertson, Images and quotients of $S O(3, \mathbb{R})$ : remarks on a theorem of van der Waerden, Rocky Mountain J. Math. 17 (1987), 1-13. 
25. W. W. Comfort and K. A. Ross, Topologies induced by groups of characters, Fund. Math. 55 (1964), $283-291$.

26. W. W. Comfort and V. Saks, Countably compact groups and finest totally bounded topologies, Pacific J. Math. 49 (1973), 33-44.

27. W. W. Comfort and F. J. Trigos-Arrieta, Remarks on a theorem of Glicksberg, General Topology and Applications (S. J. Andima, R. Kopperman, P. R. Misra, J. Z. Reichman, and A. R. Todd, eds.), Marcel Dekker, Inc., New York-Basel-Hong Kong, 1991, pp. 25-33.

28. W. W. Comfort, F. J. Trigos-Arrieta, and T.-S. Wu, The Bohr compactification, modulo a metrizable subgroup, Fund. Math. 143 (1993), 119-136.

29. , Correction to the paper "The Bohr compactification, modulo a metrizable subgroup", Fund. Math. 152 (1997), 97-98.

30. D. Dikranjan, van Douwen's problems related to the Bohr topology, Proceedings of the 9th Prague topological symposium, Prague, Czech Republic, August 19-25, 2001, Toronto: Topology Atlas, 2002, pp. 37-50.

31. D. Dikranjan, I. Prodanov, and L. Stoyanov, Topological groups: Characters, dualities, and minimal group topoligies, Pure and Applied Mathematics Vol. 130, Marcel Dekker Inc, 1990.

32. D. Dikranjan and D. Shakhmatov, Quasi-convex density and determining subgroups of compact abelian groups, J. Math. Anal. Appl. 363 (2010), 42-48.

33. _ Topological groups with many small subgroups, Topology Appl. 200 (2016), 101-132.

34. D. Dikranjan and S. Watson, A solution to van Douwen's problem on Bohr topologies, J. Pure Appl. Algebra 163 (2001), 147-158.

35. B. Efimov, On imbedding of Stone-čech compactifications of discrete spaces in bicompacta, Soviet Math. Doklady 10 (1969), 1391-1394, Russian original in: Доклады Акад. Наук СССР 187(1969), 244-266.

36. R. Engelking, General Topology, Heldermann Verlag, Berlin, 1989.

37. M. V. Ferrer and S. Hernández, Dual topologies on non-abelian groups, Topology Appl. 159 (2012), $2367-2377$.

38. M. V. Ferrer, S. Hernández, and L. Tárrega, Interpolation sets in spaces of continuous metric-valued functions, J. Math. Anal. Appl., to appear.

39. A A dichotomy property for locally compact groups, J. of Functional Analysis 275 (2018), 869-891.

40. M. V. Ferrer, S. Hernández, and V. Uspenskij, Precompact groups and property (T), J. Math. Anal. Appl. 404 (2013), $221-230$.

41. S. Gabriyelyan, Topological properties of the group of the null sequences valued in an abelian topological group, Topology Appl. 207 (2016), 136-155.

42. __ Maximally almost periodic groups and respecting properties, (2018), arXiv:1712.05521v4, 25 pages.

43. J. Galindo, Totally bounded group topologies that are Bohr topologies of LCA groups, Topology Proc. 28 (2004), $467-478$.

44. J. Galindo and S. García Ferreira, Compact groups containing dense pseudocompact subgroups without non-trivial convergent sequences, Topology Appl. 154 (2007), 476-490.

45. J Galindo and S. Hernández, Interpolation sets and the Bohr topology of locally compact groups, Adv. Math. 188 (2004), 51-68.

46. S. Garcia-Ferreira, A. Tomita, and S. Watson, Countably compact groups from a selective ultrafilter, Proc. Amer. Math. Soc. 133 (2005), 937-943.

47. I. Glicksberg, Uniform boundedness for groups, Canadian J. Math. 14 (1962), 269-276.

48. Franklin R. Gould, On certain classes of minimally almost peridic groups, Ph.D. thesis, Wesleyan University, Middletown, Connecticut, 2009.

49. J. E. Hart and K. Kunen, Bohr compactifications of discrete structures., Fund. Math. 160 (1999), $101-151$.

50. _ Bohr compactifications of non-abelian groups, Topol. Proc. 26 (2002), 593-626.

51. S. Hernández, The dimension of an LCA group in its Bohr topology, Topology Appl. 86 (1998), 63-67.

52. S. Hernández, S. Macario, and F. J. Trigos-Arrieta, Uncountable products of determined groups need not be determined, J. Math. Anal. Appl. 348 (2008), 834-842.

53. S. Hernández and F. J. Trigos-Arrieta, When a totally bounded group topology is the Bohr topology of a LCA group, (2017), submitted.

54. E. Hewitt and K. A. Ross, Abstract Harmonic Analysis: Volume I, Structure of Topological Groups, Integration Theory, Group Representations, Grundlehren der mathematischen Wissenschaften, Springer New York, 1963.

55. H. Heyer, Dualität lokalkompakter Gruppen, Springer-Verlag, Berlin-Heidelberg-New York, 1970.

56. N. Hindman, An interview with W. Wistar Comfort, http://at.yorku.ca/t/o/p/c/28.htm, 1997.

57. R. Hughes, Compactness in locally compact groups, Bull. Amer. Math. Soc. 79 (1973), $122-123$.

58. J. O. Kiltinen, Infinite Abelian groups are highly topologizable, Duke Math. J. 41 (1974), $151-154$.

59. K. Kunen, Bohr topologies and partition theorems for vector spaces, Topology Appl. 90 (1998), 97-107.

60. H. Leptin, Abelsche Gruppen mit kompakten Charaktergruppen und Dualitätstheorie gewisser linear topologischer abelscher Gruppen, Abh. Math. Seminar Univ. Hamburg 19 (1955), 244-263.

61. W. Maak, Fastperiodische Funktionen, Berlin-Heidelberg-New York, Springer-Verlag , 1967.

62. V. I. Malykhin and L. B. Shapiro, Pseudocompact groups without convergent sequences, Math. Notes 37 (1985), 59-62, Russian original in: Математические Заметки 37 (1985), 103-109.

63. A. A. Markoff, On free topological groups, C. R. (Dokl.) Acad. Sci. URSS, n. Ser. 31 (1941), $299-301$.

64. W. Moran, On almost periodic compactifications of locally compact groups, J. Lond. Math. Soc., II. Ser. 3 (1971), $507-512$.

65. E. Pearl (ed.), Open problems in topology. II, Amsterdam: Elsevier, 2007. 
66. J.-P. Pier, L'analyse harmonique. Son développement historique, Paris etc.: Masson, 1990.

67. K.-P. Podewski, Topologisierung algebraischer Strukturen, Rev. Roum. Math. Pures Appl. 22 (1977), 1283-1290.

68. I. Prodanov, Elementary example of a group without characters, Mathematics and education in mathematics, Proc. 9th Spring Conf., Sunny Beach/Bulg. 1980, 79-81 (1980).

69. S. U. Raczkowski, Totally bounded group topologies on the integers, Topology Appl. 121 (2002), 63-74.

70. Sophia U. Raczkowski-Trigos, Totally Bounded Groups, Ph.D. thesis, Wesleyan University, Middletown, Connecticut, USA, 1998.

71. D. Remus, The number of $T_{2}$-precompact group topologies on free groups, Proc. Am. Math. Soc. 95 (1985), 315 -319.

72. __ Die Anzahl von T2-präkompakten Gruppentopologien of unendlichen abelschen Gruppen, Rev. Roum. Math. Pures Appl. 31 (1986), 803-806.

73. __ Minimal and precompact group topologies on free groups, J. Pure Appl. Algebra 70 (1991), $147-157$.

74. The rôle of W. Wistar Comfort in the theory of topological groups, Topology Appl. 97 (1999), 31-49.

75. D. Remus and F. J. Trigos-Arrieta, The Bohr topology of Moore groups, Topology Appl. 97 (1999), 85-98.

76. D. Remus and M. Ursul, Van der Waerden rings, Topology Appl. 229 (2017), 148-175.

77. Dieter Remus, Zur Struktur des Verbandes der Gruppentopologien, Dissertation, Universität Hannover, Hannover (Germany), 1983.

78. _— Anzahlbestimmungen von gewissen präkompakten bzw. nicht-präkompakten hausdorffschen Gruppentopologien, Habilitationsschrift, Universität Hannover, Hannover (Germany), November 1995.

79. D. B. Shakhmatov, Imbeddings into topological groups preserving dimensions, Topology Appl. 36 (1990), $181-204$.

80. A. I. Shtern, The structure of locally bounded finite-dimensional representations of connected locally compact groups, Sb. Math. 205 (2014), 600-611, Russian original in: Матем. Сборник 205 (2014), 149-160.

81. W. Sierpiński, Sur une propriété topologique des ensembles dénombrables denses en soi, Fund. Math. 1 (1920), 11-16.

82. S. M. Sirota, The product of topological groups and extremal disconnectedness, Math. USSR Sbornik 8 (1969), 169-180, Russian original in: Матем. Сборник 79 (121) (1969), 179-192.

83. K. R. Stromberg, An elementary proof of Steinhaus's theorem, Proc. Amer. Math. Soc. 36 (1972), 308.

84. F. J. Trigos-Arrieta, Continuity, boundedness, connectedness and the Lindelöf property for topological groups, J. Pure Appl. Algebra 70 (1991), 199-210.

85. __ Pseudocompactness on groups, General Topology and Applications, Fifth Northeast Conference (S. J. Andima, R. Kopperman, P. R. Misra, J. Z. Reichman, and A. R. Todd, eds.), Marcel Dekker, Inc., New York-Basel-Hong Kong, 1991, pp. 369-378.

86. Every uncountable Abelian group admits a nonnormal group topology, Proc. Am. Math. Soc. 122 (1994), no. 3, 907-909.

87. F. Javier Trigos-Arrieta, Pseudocompactness on groups, Ph.D. thesis, Wesleyan University Middletown, Connecticut, 1991.

88. B. L. van der Waerden, Stetigkeitssätze für halbeinfache Liesche Gruppen, Math. Z. 36 (1933), $780-786$.

89. E. K. van Douwen, The maximal totally bounded group topology on $G$ and the biggest minimal G-space for Abelian groups $G$, Topology Appl. 34 (1990), 69-91.

90. Letters to W. W. Comfort, June 30, 1986 and May 9, 1987.

91. N. Th. Varopoulos, A theorem on the continuity of homomorphisms of locally compact groups, Proc. Cambridge Phil. Soc. 60 (1964), 449-463.

92. A. Weil, Sur les Espaces à Strùcture Uniforme et sur la Topologie Générale, Publ. Math. Univ. Strasbourg, Hermann, Paris, 1937.

93. _ L'intégration dans les Groupes Topologiques et ses Applications, Actualités Scientifiques et Industrielles, Publ. Math. Univ. Strasbourg, Hermann, Paris, 1951.

94. Y. Yang, Ultraproducts of quasirandom groups with small cosocles, J. Group Theory 19 (2016), $1137-1164$.

95. E. G. Zelenyuk and I. V. Protasov, Topologies on Abelian groups, Math. USSR Izvestia 37 (1991), $445-460$, Russian original in: Известия Акад. Наук СССР 54 (1990), 1090-1107.

96. Y. Zelenyuk, Topologies on Abelian Groups, Ph.D. thesis, Kyiv University, Kyiv, Ukraine, 1990.

Departmento de Matemáticas, Universitat Jaume I, 8029-AP, Castellón, Spain

E-mail address: hernande@mat.uji.es

Institut für Mathematik, Universität Paderborn, Warburger Str. 100,, D-33095 Paderborn, Germany

E-mail address: remus@math.uni-paderborn.de

Department of Mathematics, California State University, Bakersfield, Bakersfield, CA 93311

E-mail address: jtrigos@csub.edu 